DNA. Clinical findings and serological criteria confirmed the diagnosis of Juvenile SLE.

She was treated with intravenous pulse methylprednisolone, oral prednisolone acetazolamide and hydroxychloroquine. Headache and papilledema resolved however she was represented 2 weeks later with chest pain and breathlessness but on review of history she admitted to breathlessness going back several weeks, CT pulmonary angiography showed bilateral acute pulmonary embolism. Although she had thromboembolism her anticardiolipin, lupus anticoagulant, Beta 2 glycoprotein 1 antibodies were negative. Diagnosis of antiphospholipid syndrome could not be made as it did not fulfil the criteria. Anticoagulation treatment was commenced. She was also given 2 doses of Rituximab. She remains well with clinical improvement as well as improvement in her inflammatory markers.

Discussion Our patient illustrates SLE can present with idiopathic intracranial hypertension though very rare and exact pathophysiology remains unclear. Various mechanisms have been proposed including venous thrombosis, immunological or inflammatory but none have been proven. There have been few case reports in paediatric population as first presentation of lupus.

Conclusion SLE should be considered as a possible cause in a child presenting with idiopathic intracranial hypertension and a connective tissue screen should be included in the work up. In a known patient with SLE, IIH may be part of the spectrum of neuropsychiatric manifestation and should be considered as a possible cause

\section{G452(P) A COMPLEX CASE OF CYSTIC FIBROSIS AND COFFIN- SIRIS SYNDROME}

E Weir, A Devenny, P Davies. Department of Respiratory Paediatrics, Royal Hospital for Children, Glasgow, UK

\subsection{6/archdischild-2018-rcpch.441}

We describe a 4-year-old girl with advanced Cystic Fibrosis (CF) and Coffin-Siris syndrome.

Antenatally, there were concerns about Down Syndrome. She was born at term weighing $3388 \mathrm{~g}$. Dysmorphic with micrognathia, broad nasal bridge, (P)iorly rotated ears, short fingers, almond-shaped eyes with thick eyelashes and fixed bilateral talipes. Genetics for Down Syndrome negative. Admitted to Neonatal Unit with stridor and obstructive breathing requiring Vapotherm.

On newborn screening, identified as having CF (Delta F508/ $3849+10 \mathrm{kbC}>\mathrm{T}$ ) but pancreatic sufficient. Respiratory polysomnography confirmed partial obstruction with mixed events and microlaryngoscopy and bronchoscopy identified a retroverted larynx and laryngomalacia. In view of micrognathia, abnormal upper airways, obstructive breathing, and ventilatory support, required tracheostomy at 1 month. Successfully decannulated at 6 months. Significant global developmental delay and hypotonia apparent in the first few months and entered into the Decipher Developmental Disorders (DDD) study.

Frequent respiratory exacerbations and poor growth, remaining an inpatient for the first 6 months. Port-a-cath inserted at 4 months for intravenous (IV) antibiotics. High resolution computed tomography (HRCT) chest showed significant volume loss in both lower lobes, with possible associated traction bronchiectasis. Burkholderia cepacia isolated in sputum at 7 months and Pseudomonas aeruginosa at 22 months. Aged 2 years, she required home oxygen at $1 \mathrm{~L} / \mathrm{min}$. At 3 years, bronchoscopy showed thick, copious secretions throughout inflamed airways. Bronchoalveolar lavage showed no evidence of lipid-laden macrophages, but Pandorea isolated. HRCT chest significantly progressed with extensive widespread bronchiectasis, multifocal consolidation, mosaic attenuation and peripheral tree in bud opacities.

Other multisystem problems include severe gastro-oesophageal reflux, feed aversion and poor growth (weight SDS -3.09, height SDS -2.52) requiring laparoscopic fundoplication and gastrostomy at 8 months. Despite fundoplication, vomiting remains problematic. Left renal and ureteric calculi incidentally found on annual review abdominal ultrasound at 2 years requiring lithotripsy. Results from the DDD study confirmed ARID1A mutation and a diagnosis of Coffin-Siris Syndrome.

Discussion

- In a child with CF and respiratory disease out with that expected for genotype, it is important to actively seek further diagnoses.

- Coffin-Siris Syndrome is a rare multisystem disorder manifested by craniofacial abnormalities, recurrent respiratory tract infections, failure to thrive, vomiting, hypotonia, developmental delay and renal or genitourinary abnormalities.

\section{G453(P) A SURVEY OF PAEDIATRIC BRONCHOSCOPY DECONTAMINATION PRACTICE IN UK REGIONAL CENTRES}

EG Letch, EGL Batchelor, MG Semple. School of Medicine, University of Liverpool, Liverpool, UK

\subsection{6/archdischild-2018-rcpch.442}

Aims Flexible bronchoscopes are difficult to decontaminate due to their intricate internal structure. The British Thoracic Society (BTS 2013) and the Department of Health England (Heath Technical Memorandum 01-06 2016) have published guidelines on flexible endoscope decontamination.

'The Health and Social Care Act 2008: Code of Practice on the prevention and control of infections and related guidance' states that 'decontamination policy should demonstrate that it complies with guidance establishing essential quality requirements and a plan is in place for progression to best practice'. The objectives of the study were to review the practice of bronchoscope decontamination in accordance with BTS (2013) or HTM 01-06 (2013) guidelines, in UK tertiary paediatric centres and to assess for related adverse events.

Method A 10 question web-based survey was emailed to 26 paediatric tertiary centres which perform bronchoscopy in the UK. Questions considered adherence to BTS (2013) or HTM 01-06 (2013) guidelines and associated nosocomial infection between 01/01/2015-01/01/2016.

Results 11 of the 26 trusts contacted returned a completed survey.

5/11 trusts followed HTM 01-06 guidelines and 3/11 followed BTS guidelines, total 8 of 11 responding trusts.

$4(0.247 \%)$ paediatric bronchoscopies performed between $01 /$ $01 / 2015-01 / 01 / 2016$ resulted in nosocomial infection. These were all from the same centre. These infections resulted in either delayed discharge or readmission.

$2 / 11$ of the responding centres, reported sampling from the suction channel of the bronchoscope prior to each procedure 